\title{
Semigroups of Partial Transformations with Kernel and Image Restricted by an Equivalence
}

\author{
Jorge André \\ Departamento de Matemática \\ Universidade Nova de Lisboa, Portugal, jmla@ fct.unl.pt \\ Janusz Konieczny \\ Department of Mathematics, University of Mary Washington \\ Fredericksburg, VA 22401, USA, jkoniecz@umw.edu
}

\begin{abstract}
For an arbitrary set $X$ and an equivalence relation $\mu$ on $X$, denote by $P_{\mu}(X)$ the semigroup of partial transformations $\alpha$ on $X$ such that $x \mu \subseteq x(\operatorname{ker}(\alpha))$ for every $x \in \operatorname{dom}(\alpha)$, and the image of $\alpha$ is a partial transversal of $\mu$. Every transversal $K$ of $\mu$ defines a subgroup $G=G_{K}$ of $P_{\mu}(X)$. We study subsemigroups $\langle G, U\rangle$ of $P_{\mu}(X)$ generated by $G \cup U$, where $U$ is any set of elements of $P_{\mu}(X)$ of rank less than $|X / \mu|$. We show that each $\langle G, U\rangle$ is a regular semigroup, describe Green's relations and ideals in $\langle G, U\rangle$, and determine when $\langle G, U\rangle$ is an inverse semigroup and when it is a completely regular semigroup. For a finite set $X$, the top $\mathcal{J}$-class $J$ of $P_{\mu}(X)$ is a right group. We find formulas for the ranks of the semigroups $J, G \cup I, J \cup I$, and $I$, where $I$ is any proper ideal of $P_{\mu}(X)$.
\end{abstract}

2010 Mathematics Subject Classification: 20M20, 20M12, 20M17, 05E15

Keywords: Partial transformation semigroups, equivalence relations, Green's relations, regular semigroups, ideals, rank.

\section{Introduction}

In semigroup theory, transformation semigroups play a role analogous to the role of permutation groups in group theory. For a given set $X$, denote by $T(X)$ the semigroup of full transformations on $X$, that is, the set of all functions from $X$ to $X$ with function composition as the semigroup operation. (Throughout this paper, we will write functions on the right and compose from left to right; that is, for $f: A \rightarrow B$ and $g: B \rightarrow C$, we will write $x f$, rather than $f(x)$, and $x(f g)$, rather than $(g f)(x)$.) The semigroup $T(X)$ is fundamental in semigroup theory since every semigroup can be embedded in some $T(X)[7$, Theorem 1.1.2]. This result is analogous to Cayley's Theorem for groups, which states that every group can be embedded in some symmetric group $\operatorname{Sym}(X)$ of all permutations on $X$. A natural generalization of $T(X)$ is the semigroup $P(X)$ of partial transformations on $X$ (that is, functions whose domain and image are included in $X)$. The semigroup $P(X)$ contains as its subsemigroups both $T(X)$ and the symmetric inverse semigroup $\mathcal{I}(X)$ of partial injective transformations on $X$. The semigroup $\mathcal{I}(X)$ is fundamental for the important class of inverse semigroups (see [13] and [7, Chapter 5]) since every inverse semigroup can be embedded in some $\mathcal{I}(X)$ [7, Theorem 5.1.7], which is another analogue of Cayley's Theorem for groups. 
These transformation semigroups can be generalized by introducing an equivalence relation $\mu$ on $X$. In 1976, S. Madhavan [9] generalized the symmetric inverse semigroup $\mathcal{I}(X)$ this way by defining the semigroup $\mathcal{I}_{\mu}(X)$ consisting of all partial transformations $\alpha$ on $X$ such that $x \mu=x(\operatorname{ker}(\alpha))$ for every $x \in \operatorname{dom}(\alpha)$, and $\operatorname{im}(\alpha)$ is a partial transversal of $\mu$. Madhavan proved that every right normal right inverse semigroup (regular semigroup satisfying $e f g=f e g$ for all idempotents $e, f, g$ ) can be embedded into some $\mathcal{I}_{\mu}(X)$. In 2005, H. Pei [11] introduced the semigroup $T(X, \mu)$ of full transformations $\alpha$ on $X$ that preserve $\mu$ (for all $x, y \in X$, if $(x, y) \in \mu$ then $(x \alpha, y \alpha) \in \mu)$. The analogous semigroup $P(X, \mu)$ of partial transformations on $X$ preserving $\mu$ was studied in [12]. In 2004, J. Araújo and the second author [2] studied the semigroup $T(X, \mu, K)$ of full transformations $\alpha$ on $X$ that preserve both $\mu$ and a fixed transversal $K$ of $\mu(K \alpha \subseteq K)$. One can consider the analogous semigroup $P(X, \mu, K)$ of partial transformations on $X$. We also note that some subgroups of $\operatorname{Sym}(X)$, where $X$ is finite, defined by two partitions of $X$ of the same type were studied in [1].

In 2010, S. Mendes-Gonçalves and R.P. Sullivan [10] introduced the semigroup $E(X, \mu)$ of full transformations $\alpha$ on $X$ such that $\mu \subseteq \operatorname{ker}(\alpha)$, and its subsemigroup $T_{\mu}(X)$ consisting of all $\alpha \in$ $E(X, \mu)$ such that $\operatorname{im}(\alpha)$ is a partial transversal of $\mu$. They proved that $T_{\mu}(X)=\operatorname{Reg}(E(X, \mu))[10$, Theorem 2.3], where for a semigroup $S, \operatorname{Reg}(S)$ denotes the set of regular elements of $S$. Again, one can consider the analogous semigroup $E^{p}(X, \mu)$ of partial transformations $\alpha$ on $X$ such that $x \mu \subseteq x(\operatorname{ker}(\alpha))$ for every $x \in \operatorname{dom}(\alpha)$, and its subsemigroup $P_{\mu}(X)$ consisting of all $\alpha \in E^{p}(X, \mu)$ such that $\operatorname{im}(\alpha)$ is a partial transversal of $\mu$.

The semigroups described in the preceding paragraph, and others, are special cases of the sandwich semigroups studied in [4]. For non-empty sets $X$ and $Y$, denote by $\mathcal{P} \mathcal{T}_{X Y}^{a}$ the semigroup of all partial maps from $X$ to $Y$, with product $\star$ defined by $f \star g=f a g$, where $a$ is a fixed partial map from $Y$ to $X$ [4, 3.2]. The set $\operatorname{Reg}\left(\mathcal{P} \mathcal{T}_{X Y}^{a}\right)$ of regular elements of $\mathcal{P} \mathcal{T}_{X Y}^{a}$ is a subsemigroup of $\mathcal{P} \mathcal{T}_{X Y}^{a}$ [4, 3.3]. Let $\mu$ be an equivalence relation on $X$, let $K$ be a transversal of $\mu$, and let $a: X \rightarrow K$ be the function such that $k a^{-1}=k \mu$ (where $k \mu$ is the $\mu$-class of $k$ ). Then $\mathcal{P} \mathcal{T}_{K X}^{a}$ is isomorphic to $E^{p}(X, \mu)$, and $\operatorname{Reg}\left(\mathcal{P} \mathcal{T}_{K X}^{a}\right)$ is isomorphic to $P_{\mu}(X)$. Similarly, the semigroups $E(X, \mu)$ and $T_{\mu}(X)$ are special cases of the sandwich semigroups studied in [5].

For the semigroups of partial transformations mentioned above, we have

$$
\mathcal{I}_{\mu}(X) \subseteq P_{\mu}(X) \subseteq E^{p}(X, \mu) \subseteq P(X, \mu) \text { and } P(X, \mu, K) \subseteq P(X, \mu)
$$

For the corresponding full transformation semigroups, we have

$$
\left(\mathcal{I}_{\mu}(X) \cap T(X)\right) \subseteq T_{\mu}(X) \subseteq E(X, \mu) \subseteq T(X, \mu) \text { and } T(X, \mu, K) \subseteq T(X, \mu) .
$$

Let $\alpha \in P(X)$. We denote the domain of $\alpha$ by $\operatorname{dom}(\alpha)$, the image of $\alpha$ by $\operatorname{im}(\alpha)$, and the rank of $\alpha$ (the cardinality of $\operatorname{im}(\alpha)$ ) by $\operatorname{rank}(\alpha)$. The kernel of $\alpha$ is an equivalence $\operatorname{ker}(\alpha)$ on $\operatorname{dom}(\alpha)$ defined by

$$
\operatorname{ker}(\alpha)=\{(x, y) \in \operatorname{dom}(\alpha) \times \operatorname{dom}(\alpha): x \alpha=y \alpha\} .
$$

Let $\mu$ be an equivalence on $X$. For $x \in X$, we denote by $x \mu$ the $\mu$-equivalence class. Then $X / \mu=$ $\{x \mu: x \in X\}$ is the partition of $X$ induced by $\mu$. A transversal of $\mu$ is any subset $K$ of $X$ such that $K$ intersects each element of $X / \mu$ at exactly one point. Any subset of a transversal of $\mu$ is called a partial transversal of $\mu$.

Definition 1.1. Let $\mu$ be an equivalence on $X$. An $\alpha \in P(X)$ is called a $\mu$-transformation if $x \mu \subseteq$ $x(\operatorname{ker}(\alpha))$ for every $x \in \operatorname{dom}(\alpha)$, and $\operatorname{im}(\alpha)$ is a partial transversal of $\mu$. We denote by $P_{\mu}(X)$ the set of $\mu$-transformations on $X$. It is clear that $P_{\mu}(X)$ is a subsemigroup of $P(X)$. 
As we have already pointed out, $P_{\mu}(X)$ is a special case of the semigroup $\operatorname{Reg}\left(\mathcal{P} \mathcal{T}_{X Y}^{a}\right)$ studied in [4].

Definition 1.2. Let $\mu$ be an equivalence on $X$. Fix a transversal $K$ of $\mu$. An element $\sigma \in P_{\mu}(X)$ is called a $K$-permutation if $\operatorname{ker}(\sigma)=\mu$ and $\operatorname{im}(\sigma)=K$. We denote by $G_{K}$ the set of $K$-permutations. Then $G_{K}$ is a subgroup of $P_{\mu}(X)$ isomorphic to the symmetric group $\operatorname{Sym}(K)$ (see Proposition 2.1).

Notation 1.3. For the remainder of the paper, we fix a nonempty set $X$, an equivalence $\mu$ on $X$, a transversal $K$ of $\mu$, the group $G=G_{K}$, the cardinal $v=|X / \mu|=|K|$, and a nonempty set $U$ of $\mu$ transformations of rank $<v$. We denote by $\langle G, U\rangle$ the subsemigroup of $P_{\mu}(X)$ generated by the set $G \cup U$. Note that $v$ may be finite or infinite and that for every $\alpha \in P_{\mu}(X), \operatorname{rank}(\alpha) \leq v$.

The purpose of this paper is to study the semigroups $\langle G, U\rangle$. These semigroups generalize several well-known semigroups. Let $\mu=\mathrm{id}_{X}$. Then, $K=X$ is the only transversal of $\mu$ and $G=G_{K}=$ $\operatorname{Sym}(X)$. Let $X=\{1, \ldots, n\}$. Define $\alpha, \beta \in P_{\mu}(X)$ by $1 \alpha=2$ and $x \alpha=x$ for all $x \neq 1$, $\operatorname{dom}(\beta)=\{2, \ldots, n\}$ and $x \beta=x$ for all $x \in \operatorname{dom}(\beta)$. Then $\langle G,\{\alpha, \beta\}\rangle=P(X)$ [7, Exercise 1.9.13], $\langle G, \alpha\rangle=T(X)$ [7, Exercise 1.9.7], and $\langle G, \beta\rangle=\mathcal{I}(X)$ [7, Exercise 5.11.6]. For an arbitrary set $X$ and any proper ideal $I$ of $P(X), T(X)$, or $\mathcal{I}(X)$, we can select a suitable $U$ such that $\langle G, U\rangle=\operatorname{Sym}(X) \cup I$.

In Section 2, we prove that every semigroup $\langle G, U\rangle$ is regular (Theorem 2.4) and characterize the sets $U$ for which $\langle G, U\rangle$ is an inverse semigroup (Theorem 2.7) and those $U$ for which $\langle G, U\rangle$ is a completely regular semigroup (Theorem 2.10). In Section 3, we describe Green's relations (Theorems 3.1 and 3.2) and the ideals (Theorem 3.4) of $\langle G, U\rangle$, and determine the partial order of ideals of $\langle G, U\rangle$ (Theorem 3.6).

In Section 4, we assume that $X$ is a finite set. Then, every ideal of $P_{\mu}(X)$ is of the form $I_{r}=\{\alpha \in$ $\left.P_{\mu}(X): \operatorname{rank}(\alpha) \leq r\right\}$ and every $\mathcal{J}$-class of $P_{\mu}(X)$ is of the form $J_{r}=\left\{\alpha \in P_{\mu}(X): \operatorname{rank}(\alpha)=r\right\}$, where $0 \leq r \leq v$. Moreover, $J_{v}$ is a right group (Proposition 4.9), and $P_{\mu}(X)=J_{v} \cup I_{v-1}$. We find formulas for the ranks of the semigroups $G \cup I_{r}$ (Theorem 4.7), $J_{v}$ (Proposition 4.9), $J_{v} \cup I_{r}$ (Theorem 4.10), and $I_{r}$ (Corollary 4.11), where $0 \leq r<v$.

\section{Regularity}

In this section, we prove that each $\langle G, U\rangle$ is a regular semigroup and determine when $\langle G, U\rangle$ is an inverse semigroup and when it is a completely regular semigroup.

An element $a$ of a semigroup $S$ is called regular if $a=a x a$ for some $x \in S$. If all elements of $S$ are regular, we say that $S$ is a regular semigroup. An element $a^{\prime}$ in $S$ is called an inverse of $a$ in $S$ if $a=a a^{\prime} a$ and $a^{\prime}=a^{\prime} a a^{\prime}$. Since regular elements are precisely those that have inverses (if $a=a x a$ then $a^{\prime}=x a x$ is an inverse of $a$ ), we may define a regular semigroup as a semigroup in which each element has an inverse [7, p. 51]. If every element of $S$ has exactly one inverse then $S$ is called an inverse semigroup. An alternative definition is that $S$ is an inverse semigroup if it is regular and its idempotents commute [7, Theorem 5.1.1]. If every element of $S$ is in some subgroup of $S$ then $S$ is called a completely regular semigroup $[7,4.1]$.

It is well known that $P(X), T(X)$, and $\mathcal{I}(X)$ are regular semigroups. The semigroup $\mathcal{I}_{\mu}(X)$ is also regular, but $E^{p}(X, \mu)$ and $P(X, \mu)$ are not regular semigroups $[9,10,12]$.

We first prove that $G=G_{K}$ is a group.

Proposition 2.1. The set $G=G_{K}$ from Definition 1.2 is a subgroup of $P_{\mu}(X)$ isomorphic to the symmetric group $\operatorname{Sym}(K)$.

Proof. Define $f: \operatorname{Sym}(K) \rightarrow P_{\mu}(X)$ by $\delta f=\sigma$, where for every $a \in K,(a \mu) \sigma=\{a \delta\}$. Then $\operatorname{ker}(\sigma)=\mu$ and $\operatorname{im}(\sigma)=K$, so $\sigma \in G$. It is straightforward to check that $f$ is an injective semigroup homomorphism with $\operatorname{im}(f)=G$. The result follows. 
The following lemma will be crucial in our arguments. We will use it often without mentioning it explicitly.

Lemma 2.2. Let $L$ be a partial transversal of $\mu$ with $|L|<|K|$. Suppose $\sigma: L \rightarrow K$ is injective. Then $\sigma$ can be extended to $\bar{\sigma} \in G$.

Proof. Recall that $v=|K|$ and let $r=|L|$, so $r<v$. Since $\sigma$ is injective, $|L \sigma|=|L|=r<v$. Let $K^{\prime}=\{a \in K: a \in b \mu$ for some $b \in L\}, K_{1}=K \backslash K^{\prime}$, and $K_{2}=K \backslash L \sigma$. Since $L$ is a partial transversal of $\mu$, we have $\left|K^{\prime}\right|=|L|=r$. It follows that $\left|K_{1}\right|=\left|K_{2}\right|=v-r$ (if $v$ is finite) and $\left|K_{1}\right|=\left|K_{2}\right|=v$ (if $v$ is infinite). Fix a bijection $g: K_{1} \rightarrow K_{2}$.

Define $\bar{\sigma}: X \rightarrow X$ as follows. Let $x \in X$. If $x \mu \cap L=\{b\}$, then define $(x \mu) \bar{\sigma}=\{b \sigma\}$. If $x \mu \cap L=\emptyset$, then define $(x \mu) \bar{\sigma}=\{a g\}$, where $\{a\}=K_{1} \cap x \mu$. It is then clear that $\bar{\sigma} \in G$ and $\bar{\sigma}$ is an extension of $\sigma$.

Notation 2.3. Let $\alpha \in P_{\mu}(X)$ with $\operatorname{rank}(\alpha)=r$. Then $0 \leq r \leq v$. Write $\operatorname{im}(\alpha)=\left\{x_{i}\right\}_{1 \leq i \leq r}$ and let $A_{i}=x_{i} \alpha^{-1} \cap K$. We will write

$$
\alpha=\left(\begin{array}{c}
A_{i} \\
x_{i}
\end{array}\right),
$$

where it will be understood that $i$ is a cardinal ranging from 1 to $r=\operatorname{rank}(\alpha)$. This notation is justified by the fact that $\alpha \in P_{\mu}(X)$ is determined by its values on $\operatorname{dom}(\alpha) \cap K$.

For example, let $X=\{1, \ldots, 8\}, \mu$ correspond to the partition $\{\{1,2,3\},\{4,5\},\{6,7,8\}\}$, and $K=\{1,4,6\}$. Then $\alpha, \beta \in P_{\mu}(X)$ defined by $\{1,2,3,4,5\} \alpha=\{1\},\{6,7,8\} \alpha=\{4\}$, and $\{4,5,6,7,8\} \beta=\{6\}$ will be written

$$
\alpha=\left(\begin{array}{cc}
\{1,4\} & \{6\} \\
1 & 4
\end{array}\right) \text { and } \beta=\left(\begin{array}{c}
\{4,6\} \\
6
\end{array}\right) .
$$

Theorem 2.4. Each semigroup $\langle G, U\rangle$ is regular.

Proof. Let $\alpha \in\langle G, U\rangle$. If $\operatorname{rank}(\alpha)=v$, then $\alpha \in G$, and so $\alpha$ is a regular element of $\langle G, U\rangle$ since $G$ is a group. Suppose $\alpha=\left(\begin{array}{c}A_{i} \\ x_{i}\end{array}\right)$ with $\operatorname{rank}(\alpha)<v$. Fix $a_{i} \in A_{i}$ (note that $a_{i} \in K$ ) and define $\sigma:\left\{x_{i}\right\} \rightarrow K$ by $x_{i} \sigma=a_{i}$. By Lemma 2.2, $\sigma$ can be extended to $\bar{\sigma} \in G$. It is clear that $\alpha \bar{\sigma} \alpha=\alpha$, so $\alpha$ is regular.

Remark 2.5. Let $\alpha=\left(\begin{array}{c}A_{i} \\ x_{i}\end{array}\right) \in\langle G, U\rangle$ with $\operatorname{rank}(\alpha)<v$ and let $\bar{\sigma} \in G$ be as in the proof of Theorem 2.4. Since $\alpha \bar{\sigma} \alpha=\alpha$, the transformation $\alpha^{\prime}=\bar{\sigma} \alpha \bar{\sigma}$ is an inverse of $\alpha$. For this particular inverse, we have

$$
x_{i} \alpha^{\prime}=x_{i}(\bar{\sigma} \alpha \bar{\sigma})=a_{i}(\alpha \bar{\sigma})=x_{i} \bar{\sigma}=a_{i} .
$$

Theorem 2.4 is not true if we allow $U$ to contain transformations of rank $v$. For example, suppose $|X / \mu|=\aleph_{0}$ with $K=\left\{a_{1}, a_{2}, \ldots\right\}$. Consider $\alpha=\left(\begin{array}{c}A_{i} \\ a_{i}\end{array}\right)$ of rank $\aleph_{0}$ with $A_{i}=\left\{a_{2 i}\right\}$. Then $\langle G, \alpha\rangle$ is not a regular semigroup. Indeed, suppose $\alpha=\alpha \beta \alpha$ for some $\beta \in\langle G, \alpha\rangle$. Then $a_{i}=a_{2 i} \alpha=$ $a_{2 i}(\alpha \beta \alpha)=a_{i}(\beta \alpha)$, which implies $a_{i} \beta \in a_{2 i} \mu$. Thus $\operatorname{im}(\beta) \neq K$, which is impossible since $\operatorname{im}(\alpha)=$ $K$, and so the image of every element of $\langle G, \alpha\rangle$ is $K$.

However, $P_{\mu}(X)$ is a regular semigroup. We fix an idempotent $\varepsilon \in P_{\mu}(X)$ such that $\operatorname{ker}(\varepsilon)=\mu$. Since then the image of $\varepsilon$ is a transversal of $\mu$, we may assume that $\operatorname{im}(\varepsilon)=K$. Note that $\varepsilon$ is the identity of the group $G=G_{K}$ and $(a \mu) \varepsilon=\{a\}$ for every $a \in K$.

For a semigroup $S$, we denote by $\operatorname{Reg}(S)$ the set of all regular elements of $S$. 
Theorem 2.6. $P_{\mu}(X)=\operatorname{Reg}(\varepsilon P(X))$. Consequently, $P_{\mu}(X)$ is a regular semigroup.

Proof. Let $\alpha=\left(\begin{array}{c}A_{i} \\ x_{i}\end{array}\right) \in P_{\mu}(X)$. Fix $a_{i} \in A_{i}$ and consider $\beta=\left(\begin{array}{c}x_{i} \mu \\ a_{i}\end{array}\right) \in P(X)$. Then $\alpha=\varepsilon \alpha$ and $\beta=\varepsilon \beta$, so $\alpha, \beta \in \varepsilon P(X)$, and $\alpha=\alpha \beta \alpha$. Thus $\alpha \in \operatorname{Reg}(\varepsilon P(X))$.

Conversely, let $\alpha \in \operatorname{Reg}(\varepsilon P(X))$. Then, $\alpha=\alpha \beta \alpha$ for some $\beta \in \varepsilon P(X)$. By the definition of $\varepsilon$, we have $x \mu \subseteq x(\operatorname{ker}(\alpha))$ for every $x \in \operatorname{dom}(\alpha)$. Let $x, y \in \operatorname{dom}(\alpha)$ with $(x \alpha, y \alpha) \in \mu$. Then $x \alpha, y \alpha \in \operatorname{dom}(\beta)$, and so $(x \alpha) \beta=(y \alpha) \beta$ (since $z \mu \subseteq z(\operatorname{ker}(\beta))$ for every $z \in \operatorname{dom}(\beta)$ ). Thus, $x \alpha=x(\alpha \beta \alpha)=((x \alpha) \beta) \alpha=((y \alpha) \beta) \alpha=y(\alpha \beta \alpha)=y \alpha$, which implies that $\operatorname{im}(\alpha)$ is a partial transversal of $\mu$. Hence $\alpha \in P_{\mu}(X)$

We note that $\varepsilon P(X)=E^{p}(X, \mu)($ see Section 1$)$.

Theorem 2.7. A semigroup $\langle G, U\rangle$ is an inverse semigroup if and only if the following conditions are satisfied:

(a) for all $\alpha \in U$ and $x \in \operatorname{dom}(\alpha), x \mu=x(\operatorname{ker}(\alpha))$;

(b) for all $\alpha, \beta \in U, x \in \operatorname{dom}(\alpha)$, and $y \in \operatorname{dom}(\beta)$, if $(x \alpha, y \beta) \in \mu$, then $x \alpha=y \beta$.

Proof. $(\Rightarrow)$ Suppose that (a) does not hold. Then there is $\alpha=\left(\begin{array}{c}A_{i} \\ x_{i}\end{array}\right) \in U$ such that $\left|A_{j}\right| \geq 2$ for some $j$. Select $a_{i} \in A_{i}$ (for each $i$ ) and $a \in A_{j}$ such that $a \neq a_{j}$. Define $\sigma_{1}, \sigma_{2}:\left\{x_{i}\right\} \rightarrow K$ by $x_{i} \sigma_{1}=a_{i}$ for all $i, x_{i} \sigma_{2}=a_{i}$ if $i \neq j$, and $x_{j} \sigma_{2}=a$, and extend these mappings to $\overline{\sigma_{1}}, \overline{\sigma_{2}} \in G$ as in the proof of Lemma 2.2. Both $\overline{\sigma_{1}} \alpha \overline{\sigma_{1}}$ and $\overline{\sigma_{2}} \alpha \overline{\sigma_{2}}$ are inverses of $\alpha$ (see Remark 2.5). Moreover, they are distinct since $x_{j}\left(\overline{\sigma_{1}} \alpha \overline{\sigma_{1}}\right)=a_{j}$ and $x_{j}\left(\overline{\sigma_{2}} \alpha \overline{\sigma_{2}}\right)=a$. Thus $\langle G, U\rangle$ is not an inverse semigroup.

Suppose that (b) does not hold. Then there are $\alpha, \beta \in U$ such that, for some $x \in \operatorname{dom}(\alpha)$ and $y \in$ $\operatorname{dom}(\beta),(x \alpha, y \beta) \in \mu$ and $x \alpha \neq y \beta$. Let $\alpha^{\prime}$ and $\beta^{\prime}$ be inverses of $\alpha$ and $\beta$ in $\langle G, U\rangle$, respectively. Then $\alpha^{\prime} \alpha$ and $\beta^{\prime} \beta$ are idempotents. Since $(x \alpha, y \beta) \in \mu$, we have $(x \alpha) \beta^{\prime}=(y \beta) \beta^{\prime}$ and $(y \beta) \alpha^{\prime}=(x \alpha) \alpha^{\prime}$. Thus

$$
(x \alpha)\left(\alpha^{\prime} \alpha \beta^{\prime} \beta\right)=(x \alpha)\left(\beta^{\prime} \beta\right)=\left((x \alpha) \beta^{\prime}\right) \beta=\left((y \beta) \beta^{\prime}\right) \beta=y \beta .
$$

On the other hand,

$$
(x \alpha)\left(\beta^{\prime} \beta \alpha^{\prime} \alpha\right)=(y \beta)\left(\beta^{\prime} \beta \alpha^{\prime} \alpha\right)=(y \beta)\left(\alpha^{\prime} \alpha\right)=\left((y \beta) \alpha^{\prime}\right) \alpha=\left((x \alpha) \alpha^{\prime}\right) \alpha=x \alpha .
$$

Thus the idempotents $\alpha^{\prime} \alpha$ and $\beta^{\prime} \beta$ do not commute, and so $\langle G, U\rangle$ is not an inverse semigroup.

$(\Leftarrow)$ Conversely, suppose that (a) and (b) are satisfied. Note that these conditions are also satisfied by the elements of the group $G$ and they are preserved by the composition of transformations. It follows that (a) and (b) hold for all elements of $\langle G, U\rangle$. We already know that $\langle G, U\rangle$ is a regular semigroup. Let $\varepsilon, \xi \in\langle G, U\rangle$ be idempotents. We will show that $\varepsilon$ and $\xi$ commute. Note that $\alpha \in P_{\mu}(X)$ is an idempotent if and only if for all $x \in \operatorname{dom}(\alpha),(x, x \alpha) \in \operatorname{ker}(\alpha)$. Let $x \in \operatorname{dom}(\varepsilon \xi)$, that is, $x \in \operatorname{dom}(\varepsilon)$ and $x \varepsilon \in \operatorname{dom}(\xi)$. Since $\varepsilon$ satisfies (a), we have $(x, x \varepsilon) \in \mu$. Since $x \in \operatorname{dom}(\varepsilon)$ and $x \varepsilon \in \operatorname{dom}(\xi)$, it follows that $x \mu \subseteq \operatorname{dom}(\varepsilon)$ and $x \mu=(x \varepsilon) \mu \subseteq \operatorname{dom}(\xi)$. Further, by (a) applied to $\xi,(x, x \xi) \in \mu$. Thus $x \in \operatorname{dom}(\xi \varepsilon)$, so $\operatorname{dom}(\varepsilon \xi) \subseteq \operatorname{dom}(\xi \varepsilon)$. The reverse inclusion follows by symmetry, so $\operatorname{dom}(\varepsilon \xi)=$ $\operatorname{dom}(\xi \varepsilon)$. Now, both $x(\varepsilon \xi)$ and $x(\xi \varepsilon)$ are in $x \mu$. Thus, by (b), $x(\varepsilon \xi)=x(\xi \varepsilon)$. Hence the idempotents in $\langle G, U\rangle$ commute, and so $\langle G, U\rangle$ is an inverse semigroup.

Using arguments from the proof of Theorem 2.7, we can obtain the following result.

Theorem 2.8. If $|X| \geq 2$, then $P_{\mu}(X)$ and $T_{\mu}(X)$ are not inverse semigroups. 
Regarding a criterion for $\langle G, U\rangle$ to be a completely regular semigroup, we will use the following result about $P(X)$. This result has been proved for $T(X)$ [3, Theorem 2.10] and extends easily to $P(X)$.

Lemma 2.9. For every $\alpha \in P(X), \alpha$ is in a subgroup of $P(X)$ if and only if $\operatorname{im}(\alpha)$ is a transversal of $\operatorname{ker}(\alpha)$.

Theorem 2.10. A semigroup $\langle G, U\rangle$ is completely regular if and only if for every nonzero $\alpha \in U$, $\operatorname{ker}(\alpha)=X \times X$.

Proof. $(\Rightarrow)$ Suppose $\operatorname{ker}(\alpha) \neq X \times X$ for some nonzero $\alpha \in U$. Note that $\operatorname{ker}(\alpha) \neq \mu$ since $\operatorname{rank}(\alpha)<$ $v$. Thus there are two possible cases.

Case 1. $\operatorname{ker}(\alpha)=\mu \cap(\operatorname{dom}(\alpha) \times \operatorname{dom}(\alpha))$ and $\operatorname{dom}(\alpha) \neq X$.

Then there is $a \in K$ such that $a \mu \cap \operatorname{dom}(\alpha)=\emptyset$. Let $x \in \operatorname{dom}(\alpha)$ (such an $x$ exists since $\alpha \neq 0$ ) and $y=x \alpha$. Define $\sigma:\{y\} \rightarrow K$ by $y \sigma=a$ and extend it to $\bar{\sigma} \in G$. Let $\beta=\alpha \bar{\sigma} \in\langle G, U\rangle$ and note that $x \beta=a$ and $a \notin \operatorname{dom}(\beta)$. Thus $\operatorname{im}(\beta)$ is not a transversal of $\operatorname{ker}(\beta)$, and so $\langle G, U\rangle$ is not completely regular.

Case 2. $\operatorname{ker}(\alpha) \neq \mu \cap(\operatorname{dom}(\alpha) \times \operatorname{dom}(\alpha))$.

Then, since $\alpha \neq 0$, there are $x, y \in \operatorname{dom}(\alpha)$ such that $(x, y) \in \operatorname{ker}(\alpha)$ and $(x, y) \notin \mu$. If $\operatorname{im}(\alpha)$ is not a transversal of $\operatorname{ker}(\alpha)$, then $\langle G, U\rangle$ is not completely regular. Suppose $\operatorname{im}(\alpha)$ is a transversal of $\operatorname{ker}(\alpha)$. We can then assume that $x \in \operatorname{im}(\alpha)$. Let $z \in \operatorname{dom}(\alpha)$ with $z \alpha=x$ and let $w=x \alpha$.

Suppose $(x, w) \in \operatorname{ker}(\alpha)$. Then $w=x$ since $\operatorname{im}(\alpha)$ is a transversal of $\operatorname{ker}(\alpha)$. Thus $x \alpha=x=z \alpha$, and so $(x, z) \in \operatorname{ker}(\alpha)$. Since $\operatorname{ker}(\alpha) \neq X \times X$, there is $u \in X$ such that $(x, u) \notin \operatorname{ker}(\alpha)$. Suppose $u \in \operatorname{dom}(\alpha)$ and let $t=u \alpha$. Note that $t \neq x$ (since $(x, u) \notin \operatorname{ker}(\alpha)$ ), and so $(x, t) \notin \operatorname{ker}(\alpha)$ (since $x$ is the only element of $\operatorname{im}(\alpha)$ in the $\operatorname{ker}(\alpha)$-class of $x)$. Let $\{a\}=K \cap x \mu$ and $\{b\}=K \cap y \mu$. Then $a \neq b$ (since $(x, y) \notin \mu$ ). Define $\sigma_{1}:\{x, t\} \rightarrow K$ by $x \sigma_{1}=a$ and $t \sigma_{1}=b$ and extend it to $\bar{\sigma}_{1} \in G$. Let $\beta_{1}=\alpha \overline{\sigma_{1}} \in\langle G, U\rangle$ and note that $\operatorname{ker}\left(\beta_{1}\right)=\operatorname{ker}(\alpha), x \beta_{1}=a$ and $u \beta_{1}=b$. Thus $a, b \in \operatorname{im}\left(\beta_{1}\right)$ and $(x, y) \in \operatorname{ker}\left(\beta_{1}\right)$, so $\operatorname{im}\left(\beta_{1}\right)$ is not a transversal of $\operatorname{ker}\left(\beta_{1}\right)$. Suppose $u \notin \operatorname{dom}(\alpha)$ and let $\{c\}=K \cap u \mu$. Define $\sigma_{2}:\{x\} \rightarrow K$ by $x \sigma_{2}=c$ and extend it to $\overline{\sigma_{2}} \in G$. Let $\beta_{2}=\alpha \overline{\sigma_{2}} \in\langle G, U\rangle$ and note that $\operatorname{ker}\left(\beta_{2}\right)=\operatorname{ker}(\alpha), x \beta_{2}=c$, and $c \notin \operatorname{dom}\left(\beta_{2}\right)$. Thus $\operatorname{im}\left(\beta_{2}\right)$ is not a transversal of $\operatorname{ker}\left(\beta_{2}\right)$. Hence, when $(x, w) \in \operatorname{ker}(\alpha),\langle G, U\rangle$ is not completely regular.

Finally, suppose $(x, w) \notin \operatorname{ker}(\alpha)$. Let $\{a\}=K \cap x \mu$ and $\{b\}=K \cap y \mu$. Then $a \neq b$ (since $(x, y) \notin$ $\mu$ ). Define $\sigma:\{x, w\} \rightarrow K$ by $x \sigma=a$ and $w \sigma=b$ and extend it to $\bar{\sigma} \in G$. Let $\beta=\alpha \bar{\sigma} \in\langle G, U\rangle$ and note that $\operatorname{ker}(\beta)=\operatorname{ker}(\alpha), z \beta=a$ and $x \beta=b$. Thus $a, b \in \operatorname{im}(\beta)$ and $(x, y) \in \operatorname{ker}(\beta)$, so $\operatorname{im}(\beta)$ is not a transversal of $\operatorname{ker}(\beta)$. Hence, when $(x, w) \notin \operatorname{ker}(\alpha),\langle G, U\rangle$ is not completely regular.

$(\Leftarrow)$ Conversely, suppose $\operatorname{ker}(\alpha)=X \times X$ for every nonzero $\alpha \in U$. Then, every element of $\langle G, U\rangle$ is either an element of the group $G$ or a constant idempotent with domain $X$. Thus $\langle G, U\rangle$ is completely regular.

Using arguments from the proof of Theorem 2.10, we can obtain the following result.

Theorem 2.11. If $\mu \neq X \times X$, then $P_{\mu}(X)$ and $T_{\mu}(X)$ are not completely regular semigroups.

\section{Green's relations and ideals}

In this section, we determine Green's relations and ideals in $\langle G, U\rangle$.

Let $S$ be a semigroup and denote by $S^{1}$ the semigroup $S$ with an identity adjoined (if necessary). Then, for every $a \in S, S^{1} a, a S^{1}$, and $S^{1} a S^{1}$ are, respectively, the principal left ideal, principal right 
ideal, and principal ideal generated by $a$. The principal ideals of $S$ have been used to define five equivalence relations on $S$ that are among the most important tools in studying semigroups. For $a, b \in S$, we say that $a \mathcal{L} b$ if $S^{1} a=S^{1} b, a \mathcal{R} b$ if $a S^{1}=b S^{1}$, and $a \mathcal{J} b$ if $S^{1} a S^{1}=S^{1} b S^{1}$. We define $\mathcal{H}$ as the intersection of $\mathcal{L}$ and $\mathcal{R}$, and $\mathcal{D}$ as the join of $\mathcal{L}$ and $\mathcal{R}$, that is, the smallest equivalence relation on $S$ containing both $\mathcal{L}$ and $\mathcal{R}$. These equivalences are called Green's relations. The relations $\mathcal{L}$ and $\mathcal{R}$ commute [7, Proposition 2.1.3], and consequently $\mathcal{D}=\mathcal{L} \circ \mathcal{R}=\mathcal{R} \circ \mathcal{L}$. For a Green relation $\mathcal{F}$ in $S$ and $a \in S$, we denote by $F_{a}$ the $\mathcal{F}$-equivalence class of $a$.

Green's relations in the semigroup $P(X)$ are well known: $\alpha \mathcal{L} \beta \Leftrightarrow \operatorname{im}(\alpha)=\operatorname{im}(\beta) ; \alpha \mathcal{R} \beta \Leftrightarrow$ $\operatorname{ker}(\alpha)=\operatorname{ker}(\beta) ; \alpha \mathcal{J} \beta \Leftrightarrow \operatorname{rank}(\alpha)=\operatorname{rank}(\beta)$, and $\mathcal{D}=\mathcal{J}$.

If $T$ is a regular subsemigroup of $S$ and $\mathcal{F} \in\{\mathcal{L}, \mathcal{R}, \mathcal{H}\}$, then $\mathcal{F}$ in $T$ is the restriction of $\mathcal{F}$ in $S$ to $T \times T$ [7, Proposition 2.4.2]. Thus, by Theorem 2.4, we have the following result.

Theorem 3.1. For all $\alpha, \beta \in\langle G, U\rangle, \alpha \mathcal{L} \beta \Leftrightarrow \operatorname{im}(\alpha)=\operatorname{im}(\beta)$ and $\alpha \mathcal{R} \beta \Leftrightarrow \operatorname{ker}(\alpha)=\operatorname{ker}(\beta)$.

The corresponding statements about relations $\mathcal{D}$ and $\mathcal{J}$ in a regular subsemigroup $T$ of a semigroup $S$ are not true. Therefore, the next result requires a proof. First we note that in any semigroup $S$, the inclusion relation on the set of principal ideals induces the partial order relation $\leq$ on the set of $\mathcal{J}$-classes:

$$
J_{a} \leq J_{b} \Leftrightarrow S^{1} a S^{1} \subseteq S^{1} b S^{1} .
$$

Theorem 3.2. In every $\langle G, U\rangle, \mathcal{D}=\mathcal{J}$. Moreover, if $\alpha, \beta \in\langle G, U\rangle$, then $J_{\alpha} \leq J_{\beta} \Leftrightarrow \operatorname{rank}(\alpha) \leq$ $\operatorname{rank}(\beta)$. Consequently, $\alpha \mathcal{J} \beta \Leftrightarrow \operatorname{rank}(\alpha)=\operatorname{rank}(\beta)$.

Proof. Let $\alpha, \beta \in\langle G, U\rangle$. If $J_{\alpha} \leq J_{\beta}$, then $\alpha=\gamma_{1} \beta \gamma_{2}$ for some $\gamma_{1}, \gamma_{2} \in\langle G, U\rangle^{1}$, which implies $\operatorname{rank}(\alpha) \leq \operatorname{rank}(\beta)$. Conversely, let $r=\operatorname{rank}(\alpha), t=\operatorname{rank}(\beta)$, and suppose $r \leq t$. If $r=t=v$, then $\alpha, \beta \in G$, and so, since $G$ is a group, $\alpha \mathcal{J} \beta$, which implies $J_{\alpha} \leq J_{\beta}$. Suppose $r<v$. Let $\alpha=\left(\begin{array}{l}A_{i} \\ x_{i}\end{array}\right)$ and $\beta=\left(\begin{array}{c}B_{j} \\ y_{j}\end{array}\right)$, with $1 \leq i \leq r$ and $1 \leq j \leq t$. Fix $b_{j} \in B_{j}$ and define $\sigma_{1}:\left\{x_{i}\right\} \rightarrow\left\{b_{i}\right\}$ by $x_{i} \sigma_{1}=b_{i}$ $\left(1 \leq i \leq r\right.$ ). Then $\sigma_{1}$ is well defined (since $r \leq t$ ) and injective. Thus, since $r<v, \sigma_{1}$ can be extended to $\overline{\sigma_{1}} \in G$. For every $1 \leq i \leq r$, let $\left\{c_{i}\right\}=x_{i} \mu \cap K$, and define $\sigma_{2}:\left\{y_{i}\right\}_{1 \leq i \leq r} \rightarrow\left\{c_{i}\right\}$ by $y_{i} \sigma_{2}=c_{i}$, and extend $\sigma_{2}$ to $\overline{\sigma_{2}} \in G$. Let $\alpha^{\prime} \in\langle G, U\rangle$ be an inverse of $\alpha$. Then, for all $1 \leq i \leq r$ and all $a_{i} \in A_{i}$,

$$
\begin{aligned}
a_{i}\left(\alpha \overline{\sigma_{1}} \beta \overline{\sigma_{2}} \alpha^{\prime} \alpha\right) & =x_{i}\left(\overline{\sigma_{1}} \beta \overline{\sigma_{2}} \alpha^{\prime} \alpha\right)=b_{i}\left(\beta \overline{\sigma_{2}} \alpha^{\prime} \alpha\right)=y_{i}\left(\overline{\sigma_{2}} \alpha^{\prime} \alpha\right) \\
& =c_{i}\left(\alpha^{\prime} \alpha\right)=x_{i}\left(\alpha^{\prime} \alpha\right)=a_{i}\left(\alpha \alpha^{\prime} \alpha\right)=a_{i} \alpha
\end{aligned}
$$

It follows that $\alpha=\left(\alpha \overline{\sigma_{1}}\right) \beta\left(\overline{\sigma_{2}} \alpha^{\prime} \alpha\right)$, and so $J_{\alpha} \leq J_{\beta}$.

In every semigroup, $\mathcal{D} \subseteq \mathcal{J}$. Let $\alpha, \beta \in\langle G, U\rangle$ with $\alpha \mathcal{J} \beta$. Then, by the first part of the proof, $\operatorname{rank}(\alpha)=\operatorname{rank}(\beta)$. Let $r=\operatorname{rank}(\alpha)$. If $r=v$, then $\alpha, \beta \in G$, and so $\alpha \mathcal{D} \beta$. Suppose $r<v$. Let $\alpha=\left(\begin{array}{c}A_{i} \\ x_{i}\end{array}\right)$, and $\beta=\left(\begin{array}{c}B_{i} \\ y_{i}\end{array}\right)$, with $1 \leq i \leq r$. Fix $a_{i} \in A_{i}$ and $b_{i} \in B_{i}$, define $\sigma_{1}:\left\{x_{i}\right\} \rightarrow\left\{a_{i}\right\}$ by $x_{i} \sigma_{1}=a_{i}, \sigma_{2}:\left\{a_{i}\right\} \rightarrow\left\{b_{i}\right\}$ by $a_{i} \sigma_{2}=b_{i}$, and extend $\sigma_{1}$ to $\overline{\sigma_{1}} \in G$ and $\sigma_{2}$ to $\overline{\sigma_{2}} \in G$. Let $\gamma=\alpha \overline{\sigma_{1}} \overline{\sigma_{2}} \beta \in\langle G, U\rangle$. Then, for all $1 \leq i \leq r$ and all $c_{i} \in A_{i}$,

$$
c_{i} \gamma=c_{i}\left(\alpha \overline{\sigma_{1}} \overline{\sigma_{2}} \beta\right)=a_{i}\left(\alpha \overline{\sigma_{1}} \overline{\sigma_{2}} \beta\right)=x_{i}\left(\overline{\sigma_{1}} \overline{\sigma_{2}} \beta\right)=a_{i}\left(\overline{\sigma_{2}} \beta\right)=b_{i} \beta=y_{i}
$$

It follows that $\operatorname{ker}(\gamma)=\operatorname{ker}(\alpha)$ and $\operatorname{im}(\gamma)=\operatorname{im}(\beta)$. Thus, by Theorem 3.1, $\alpha \mathcal{R} \gamma$ and $\gamma \mathcal{L} \beta$, and so $\alpha \mathcal{D} \beta$ since $\mathcal{D}=\mathcal{R} \circ \mathcal{L}$. Hence $\mathcal{J} \subseteq \mathcal{D}$, and so $\mathcal{D}=\mathcal{J}$. 
Notation 3.3. Let $\beta \in\langle G, U\rangle$ with $r=\operatorname{rank}(\beta)$. By Theorem 3.2, the principal ideal of $\langle G, U\rangle$ generated by $\beta$ consists of all $\alpha \in\langle G, U\rangle$ such that $\operatorname{rank}(\alpha) \leq r$. We denote this principal ideal by $I_{r}$, that is,

$$
I_{r}=\{\alpha \in\langle G, U\rangle: \operatorname{rank}(\alpha) \leq r\} .
$$

For a cardinal $k$, we denote by $k^{+}$the successor cardinal of $k$ [8, p. 162]. For $1 \leq r \leq v^{+}$, let

$$
E_{r}=\{\alpha \in\langle G, U\rangle: \operatorname{rank}(\alpha)<r\} .
$$

It is clear that $E_{r}$ is an ideal of $\langle G, U\rangle$. Note that $E_{v^{+}}=\langle G, U\rangle$ and that for every $0 \leq r \leq v, I_{r}=E_{r^{+}}$.

We will now prove that every ideal of $\langle G, U\rangle$ is equal to some ideal $E_{r}$, where $1 \leq r \leq v^{+}$, and determine the partial order of ideals of $\langle G, U\rangle$.

Theorem 3.4. Let $I$ be an ideal of $\langle G, U\rangle$. Then $I=E_{r}$ for some $r$ with $1 \leq r \leq v^{+}$.

Proof. Let $r$ be the minimum cardinal such that $r \leq v^{+}$and $\operatorname{rank}(\beta)<r$ for every $\beta \in I$. (Such an $r$ exists because $\operatorname{rank}(\beta)<v^{+}$for every $\beta \in\langle G, U\rangle$.) Clearly, $I \subseteq E_{r}$. Let $\alpha \in E_{r}$. By the minimality of $r$, there is $\beta \in I$ such that $\operatorname{rank}(\alpha) \leq \operatorname{rank}(\beta)$. By Theorem 3.2, $\alpha=\gamma_{1} \beta \gamma_{2}$ for some $\gamma_{1}, \gamma_{2} \in\langle G, U\rangle^{1}$. Thus $\alpha \in I$, and so $E_{r} \subseteq I$.

It follows from Theorem 3.4 that the ideals of every semigroup $\langle G, U\rangle$ form a chain. To describe this chain, we need the following lemma.

Lemma 3.5. Let $t<v$ and suppose $U$ contains some $\alpha$ with $\operatorname{rank}(\alpha)=t$. Then:

(1) for every cardinal $r$ with $1 \leq r<t$, there is $\beta \in\langle G, U\rangle$ such that $\operatorname{rank}(\beta)=r$;

(2) if $U$ contains some $\gamma$ with $\operatorname{dom}(\gamma) \neq X$, then $0 \in\langle G, U\rangle$.

Proof. Let $\alpha=\left(\begin{array}{c}A_{i} \\ x_{i}\end{array}\right)$, where $1 \leq i \leq t$, and fix $a_{i} \in A_{i}$. To prove (1), let $1 \leq r<t$. Suppose $t \geq \aleph_{0}$ and consider two possible cases.

Case 1. There is a cardinal $l$ with $1 \leq l \leq t$ such that $\left|A_{l}\right|>t$.

Then, there is a subset $\left\{b_{j}\right\}_{r<j \leq t}$ of $A_{l}$ such that each $b_{j} \neq a_{l}$ and $b_{j_{1}} \neq b_{j_{2}}$ if $j_{1} \neq j_{2}$. If $l \leq r$, then define $\sigma:\left\{x_{i}\right\} \rightarrow K$ by $x_{i} \sigma=a_{i}$ if $1 \leq i \leq r$, and $x_{i} \sigma=b_{i}$ if $r<i \leq t$; if $l>r$, then define $\sigma:\left\{x_{i}\right\} \rightarrow K$ by $x_{i} \sigma=a_{i}$ if $1 \leq i<r, x_{r} \sigma=a_{l}$, and $x_{i} \sigma=b_{i}$ if $r<i \leq t$. In either case, we can extend $\sigma$ to $\bar{\sigma} \in G$. Let $\beta=\alpha \bar{\sigma} \alpha \in\langle G, U\rangle$. Then, $\operatorname{im}(\beta)=\left\{x_{i}\right\}_{1 \leq i \leq r}$ if $l \leq r$, and $\operatorname{im}(\beta)=\left\{x_{i}\right\}_{1 \leq i<r} \cup\left\{x_{l}\right\}$ if $l>r$. In either case, $\operatorname{rank}(\beta)=r$.

Case 2. For every cardinal $i$ with $1 \leq i \leq t,\left|A_{i}\right| \leq t$.

Then, $\left|\bigcup A_{i}\right| \leq t \cdot t=t<v$, and so there is a subset $\left\{b_{j}\right\}_{r<j \leq t}$ of $K \backslash \bigcup A_{i}$ such that $b_{j_{1}} \neq b_{j_{2}}$ if $j_{1} \neq j_{2}$. Define $\sigma:\left\{x_{i}\right\} \rightarrow K$ by $x_{i} \sigma=a_{i}$ if $1 \leq i \leq r$, and $x_{i} \sigma=b_{i}$ if $r<i \leq t$. Then, for $\beta=\alpha \bar{\sigma} \alpha \in\langle G, U\rangle$, we have $\operatorname{im}(\beta)=\left\{x_{i}\right\}_{1 \leq i \leq r}$, and $\operatorname{so} \operatorname{rank}(\beta)=r$.

Suppose $t<\aleph_{0}$. We may assume that $r=t-1$. (The result will then follow by an inductive argument.) Suppose there is $l \in\{1, \ldots, t\}$ such that $\left|A_{l}\right| \geq 2$. We then have some $b \in A_{l}$ with $b \neq a_{l}$. Select $j \in\{1, \ldots, t\}$ with $j \neq l$ (possible since $1 \leq r<t$, so $t \geq 2$ ). Define $\sigma:\left\{x_{i}\right\} \rightarrow K$ by $x_{i} \sigma=a_{i}$ if $i \neq j$, and $x_{j} \sigma=b$, and extend $\sigma$ to $\bar{\sigma} \in G$. Then, for $\beta=\alpha \bar{\sigma} \alpha \in\langle G, U\rangle$, we have $\operatorname{im}(\beta)=\left\{x_{i}\right\}_{i \neq j}$, and so $\operatorname{rank}(\beta)=t-1$. Suppose $\left|A_{i}\right|=1$ for all $i$. Since $t<v$, we then have some $b \in K \backslash \bigcup_{1 \leq i \leq t} A_{i}$. Define $\sigma:\left\{x_{i}\right\} \rightarrow K$ by $x_{i} \sigma=a_{i}$ if $i<t$ and $x_{t} \sigma=b$, and extend $\sigma$ to $\bar{\sigma} \in G$. Then, for $\beta=\alpha \bar{\sigma} \alpha \in\langle G, U\rangle$, we have $\operatorname{im}(\beta)=\left\{x_{i}\right\}_{i \neq t}$, and so $\operatorname{rank}(\beta)=t-1$.

To prove (2), suppose $U$ contains some $\gamma$ with $\operatorname{dom}(\gamma) \neq X$. Select $a \in K$ such that $a \notin \operatorname{dom}(\gamma)$. By (1), there exists $\beta \in\langle G, U\rangle$ with $\operatorname{rank}(\beta)=1$. Let $\operatorname{im}(\beta)=\{y\}$. Define $\sigma:\{y\} \rightarrow K$ by $y \sigma=a$ and extend $\sigma$ to $\bar{\sigma} \in G$. Then $\beta \bar{\sigma} \gamma=0$. 
For sets $A$ and $B$, we will write $A \subset B$ to mean $A \subseteq B$ and $A \neq B$.

Theorem 3.6. Let $m=\min \{r: \operatorname{rank}(\alpha)<r$ for every $\alpha \in U\}$. Then:

(1) if $U$ consists of full transformations on $X$, then the chain of ideals of $\langle G, U\rangle$ is isomorphic to the chain of cardinals $\{r: 2 \leq r \leq m\} \cup\left\{v^{+}\right\}$;

(2) if $U$ contains a strictly partial transformation on $X$, then the chain of ideals of $\langle G, U\rangle$ is isomorphic to the chain of cardinals $\{r: 1 \leq r \leq m\} \cup\left\{v^{+}\right\}$.

Proof. Note that $m \leq v$. Suppose $U$ consists of full transformations on $X$. Let $2 \leq r<m$. By the minimality of $m$, there is $\alpha \in U$ such that $r \leq \operatorname{rank}(\alpha)$. By Lemma 3.5, $\langle G, U\rangle$ contains a transformation of rank $<r$. Thus, for every cardinal $r$ with $2 \leq r<m, E_{r} \neq \emptyset$. Moreover, $E_{m} \neq \emptyset$ (since $E_{2} \subseteq E_{m}$ ) and $E_{1}=\emptyset$ (since 0 cannot be a product of full transformations on $X$ ). Hence, by Theorem 3.4, $\left\{E_{r}: 2 \leq r \leq m\right\} \cup\left\{E_{v^{+}}\right\}$is the set of ideals of $\langle G, U\rangle$. Define

$$
f:\{r: 2 \leq r \leq m\} \cup\left\{v^{+}\right\} \rightarrow\left\{E_{r}: 2 \leq r \leq m\right\} \cup\left\{E_{v^{+}}\right\}
$$

by $r f=E_{r}(2 \leq r \leq m)$ and $v^{+} f=E_{v^{+}}$. It is then clear that $f$ is surjective and that it preserves the order (for all $s, t \in\{r: 2 \leq r \leq m\} \cup\left\{v^{+}\right\}$, if $s \leq t$, then $E_{s} \subseteq E_{t}$ ). Let $s, t \in\{r: 2 \leq r \leq m\} \cup\left\{v^{+}\right\}$ with $s<t$. If $t=v^{+}$, then $E_{s} \subset E_{t}$ since $G \subseteq E_{v^{+}}$and $G \cap E_{s}=\emptyset$. Suppose $t \leq m$. By the minimality of $m, U$ contains $\alpha$ such that $s \leq \operatorname{rank}(\alpha)$. If $\operatorname{rank}(\alpha)<t$, then $\alpha \in E_{t} \backslash E_{s}$, so $E_{s} \subset E_{t}$. Suppose $\operatorname{rank}(\alpha) \geq t$. Then, by Lemma 3.5, there is $\beta \in\langle G, U\rangle$ with $\operatorname{rank}(\beta)=s$. Thus $\beta \in E_{t} \backslash E_{s}$, so $E_{s} \subset E_{t}$. Hence $f$ is injective, and so it is a poset isomorphism.

We have proved (1). The proof of (2) is almost identical. The difference is that, if $U$ contains a strictly partial transformation, then $E_{1} \neq \emptyset$ by Lemma 3.5 .

For example, denote by $\mathbb{Z}, \mathbb{Q}$, and $\mathbb{R}$, the sets of integers, rational numbers, and real numbers, respectively. Let $X=\mathbb{R}$, let $\mu$ be defined by the partition

$$
\{\{-n, n\}: n \in \mathbb{Z}\} \cup\{\mathbb{Q} \backslash \mathbb{Z}\} \cup\{\{x\}: x \in \mathbb{R} \backslash \mathbb{Q}\},
$$

and $K=\{0,1,2, \ldots\} \cup\left\{\frac{1}{2}\right\} \cup\{x: x \in \mathbb{R} \backslash \mathbb{Q}\}$. Then $v=2^{\aleph_{0}}$. Consider the idempotent

$$
\varepsilon=\left(\begin{array}{cccccc}
\{0\} & \{1\} & \{2\} & \ldots & \left\{\frac{1}{2}\right\} & K \backslash \mathbb{Q} \\
0 & 1 & 2 & \ldots & \frac{1}{2} & \sqrt{2}
\end{array}\right) \in P_{\mu}(\mathbb{R})
$$

(see Notation 2.3) and the semigroup $\langle G, \varepsilon\rangle$. Note that $\varepsilon$ is a full transformation on $\mathbb{R}$ and that $\operatorname{rank}(\varepsilon)=$ $\aleph_{0}$. Thus, the cardinal $m$ from Theorem 3.6 is $\aleph_{1}$ (see [8, p. 131]) and the chain of ideals of $\langle G, \varepsilon\rangle$ is isomorphic to the chain of cardinals

$$
2<3<4<\ldots<\aleph_{0}<\aleph_{1}<\left(2^{\aleph_{0}}\right)^{+},
$$

which, in turn, is isomorphic to the ordinal $\omega_{0}+2$ (see [8, p. 131]).

\section{Ranks}

Throughout this section, $X$ will be a finite set. By the more general results obtained in [4, 3.4], we can conclude the following. Every ideal of $P_{\mu}(X)$ is of the form $E_{s}=\left\{\alpha \in P_{\mu}(X): \operatorname{rank}(\alpha)<s\right\}$, where $1 \leq s \leq v^{+}$. For a finite set $X, E_{s}=I_{r}=\left\{\alpha \in P_{\mu}(X): \operatorname{rank}(\alpha) \leq r\right\}$, where $r=s-1$. Thus 
$\left\{I_{r}: 0 \leq r \leq v\right\}$ is the set of ideals of $P_{\mu}(X)$. Each ideal $I_{r}$ is principal and is generated by any $\alpha \in P_{\mu}(X)$ of rank $r$. Moreover, $I_{v}=P_{\mu}(X)$ and if $r<v$, then $I_{r}$ is a proper ideal of $P_{\mu}(X)$. Let $J_{r}$ be the set of elements of $P_{\mu}(X)$ of rank $r$, where $0 \leq r \leq v$. Then $\left\{J_{r}: 0 \leq r \leq v\right\}$ is the set of $\mathcal{J}$-classes of $P_{\mu}(X)$, with $J_{0}<J_{1}<\ldots<J_{v}$.

Since $v$ is finite, $J_{v}$ is the union of groups $G_{M}$, where $M$ ranges over all transversals of $\mu$ (see Definition 1.2). We will show that $J_{v}$ is a right group (Proposition 4.9).

In this section, we find formulas for the ranks of the semigroups $G \cup I_{r}, J_{v}, J_{v} \cup I_{r}$, and $I_{r}$, where $0 \leq r<v$. (For $r=v-1$, we have $J_{v} \cup I_{r}=P_{\mu}(X)$.) We also record the corresponding formulas for $T_{\mu}(X)=P_{\mu}(X) \cap T(X)$.

Definition 4.1. Let $S$ be a semigroup. The $\operatorname{rank}$ of $S$, denoted $\operatorname{rank} S$, is the minimum cardinality of a generating set of $S$.

The ranks of various transformation semigroups have been found. For example, for a finite set $X$, $\operatorname{rank} P(X)=4$, rank $T(X)=3$, and $\operatorname{rank} \mathcal{I}(X)=3$. The following general result for the ranks of finite semigroups proved in [6] is useful when working with transformation semigroups.

Lemma 4.2. ([6, Theorem 10]) Let $S$ be a finite nontrivial semigroup with a maximal regular class $\mathcal{J}$-class $J$ such that $\langle J\rangle=S$. Suppose that each group $\mathcal{H}$-class of $J$ has rank $\leq 2$, and it is not the case that $J$ has exactly one idempotent in every $\mathcal{R}$-class and in every $\mathcal{L}$-class. Then $\operatorname{rank} S=\max \left\{m_{l}, m_{r}\right\}$, where $m_{l}$ and $m_{r}$ are the numbers of $\mathcal{L}$ - and $\mathcal{R}$-classes in $J$, respectively.

Definition 4.3. Let $\alpha=\left(\begin{array}{c}A_{i} \\ x_{i}\end{array}\right) \in P_{\mu}(X)$ with $\operatorname{rank}(\alpha)=r, 0 \leq r \leq v$, as in Notation 2.3. In this section, we will always assume that $\left|A_{1}\right| \geq\left|A_{2}\right| \geq \ldots \geq\left|A_{r}\right|$. Let $n_{i}=\left|A_{i}\right|$ and $m=\left|K \backslash \bigcup_{1 \leq i \leq r} A_{i}\right|$. Then the sequence

$$
\left(n_{1}, n_{2}, \ldots, n_{r} ; m\right)
$$

will be called the $\mu$-type of $\alpha$ and denoted type ${ }_{\mu}(\alpha)$. We will call the number $m$ the deficit of $\alpha$. Note that $n_{1}+n_{2}+\cdots+n_{r}+m=v$ and that if $\alpha \neq 0$, then the sequence $\left(n_{1}, n_{2}, \ldots, n_{r}\right)$ is a partition of $v-m$ with $r$ parts [14, p. 235].

By a $\mu$-type we will mean any sequence $\left(n_{1}, n_{2}, \ldots, n_{r} ; m\right)$ with $0 \leq r \leq v$, each $n_{i} \geq 1, m \geq 0$, and $n_{1}+n_{2}+\cdots+n_{r}+m=v$.

For example, every $\sigma \in G$ has $\mu$-type $(1,1, \ldots, 1 ; 0)$. Let $X=\{1, \ldots, 9\}, \mu$ be defined by the partition $\{\{1,2,3\},\{4,5\},\{6,7,8\},\{9\}\}$, and $K=\{1,4,6,9\}$. Then

$$
\alpha=\left(\begin{array}{cc}
\{1,4\} & \{6\} \\
1 & 2
\end{array}\right) \in P_{\mu}(X)
$$

has $\mu$-type $(2,1 ; 1)$.

Lemma 4.4. Let $\alpha, \beta \in P_{\mu}(X)$ with $\operatorname{rank}(\alpha)=\operatorname{rank}(\beta)=r<v$. Then:

(1) $\operatorname{type}_{\mu}(\sigma \beta)=\operatorname{type}_{\mu}(\beta)$ for all $\sigma \in P_{\mu}(X)$ with $\operatorname{rank}(\sigma)=v$;

(2) if $\alpha=\beta \gamma$, for some $\gamma \in P_{\mu}(X)$, then type ${ }_{\mu}(\alpha)=\operatorname{type}_{\mu}(\beta)$.

Proof. Let $\alpha=\left(\begin{array}{c}A_{i} \\ x_{i}\end{array}\right)$ and $\beta=\left(\begin{array}{c}B_{i} \\ y_{i}\end{array}\right), 1 \leq i \leq r$. Then for every $\sigma \in P_{\mu}(X)$ with $\operatorname{rank}(\sigma)=v$, $\sigma \beta=\left(\begin{array}{c}B_{i} \sigma^{-1} \\ y_{i}\end{array}\right)$, where $B_{i} \sigma^{-1}=\left\{k \in K: k \sigma \in b \mu\right.$ for some $\left.b \in B_{i}\right\}$. Since $\sigma$ maps different 
elements of $K$ to elements in different $\mu$-classes, $\left|B_{i} \sigma^{-1}\right|=\left|B_{i}\right|$ for all $i$, so $\sigma \beta$ and $\beta$ have the same $\mu$-type.

Suppose $\alpha=\beta \gamma$ for some $\gamma \in P_{\mu}(X)$. Let $b \in B_{i}$. Then either $b \in A_{j}$, for some $j$, or $b \in$ $K \backslash \bigcup_{1 \leq i \leq r} A_{i}$. Suppose $b \in A_{j}$ and let $c \in B_{i}$. Then $b \beta=c \beta$, and so $x_{j}=b \alpha=b(\beta \gamma)=c(\beta \gamma)=c \alpha$. Thus $c \in A_{j}$.

We have proved that for every $i$, either $B_{i} \subseteq A_{j}$, for some $j$, or $B_{i} \subseteq K \backslash \bigcup_{1 \leq i \leq r} A_{i}$. Let $j \in$ $\{1, \ldots, r\}$ and $a \in A_{j}$. Then $a \in \operatorname{dom}(\alpha)$, and so $a \in \operatorname{dom}(\beta)$ (since $\alpha=\beta \gamma$ ). Thus $a \in B_{i}$ for some $i$, and so, by the foregoing argument, $a \in B_{i} \subseteq A_{j}$.

It then follows that every $A_{j}$ is a union of some distinct $B_{i_{1}}, \ldots, B_{i_{k_{j}}}$. But the number of $A_{j} s$ is $r$ and the number of $B_{i} s$ is also $r$. Hence each $k_{j}$ must equal to 1 , that is, for every $j$, there is $i_{j}$ such that $A_{j}=B_{i_{j}}$, and $i_{j_{1}} \neq i_{j_{2}}$ if $j_{1} \neq j_{2}$. It follows that type ${ }_{\mu}(\alpha)=\operatorname{type}_{\mu}(\beta)$.

The following proposition will be crucial for the rank results.

Proposition 4.5. Let $0 \leq r<v$ and suppose $U \subseteq J_{r}$, where $J_{r}$ is the $\mathcal{J}$-class of $P_{\mu}(X)$ of rank $r$. Then $J_{r} \subseteq\langle G, U\rangle$ if and only if for every $\mu$-type $\left(n_{1}, n_{2}, \ldots, n_{r} ; m\right)$ and every partial transversal $L$ of $\mu$ with $|L|=r$ and $L \nsubseteq K$, there are $\alpha, \beta \in U$ such that type ${ }_{\mu}(\alpha)=\left(n_{1}, n_{2}, \ldots, n_{r} ; m\right)$ and $\operatorname{im}(\beta)=L$.

Proof. Suppose $J_{r} \subseteq\langle G, U\rangle$. Let $\left(n_{1}, n_{2}, \ldots, n_{r} ; m\right)$ be a $\mu$-type and $L$ be a partial transversal of $\mu$ with $|L|=r$ and $L \nsubseteq K$. Since $J_{r} \subseteq\langle G, U\rangle$, there is $\eta \in\langle G, U\rangle$ such that type ${ }_{\mu}(\eta)=\left(n_{1}, n_{2}, \ldots, n_{r} ; m\right)$ and $\operatorname{im}(\eta)=L$. Since $\langle G, U\rangle$ is generated by $G \cup U, \eta=\sigma \alpha \gamma$ or $\eta=\alpha \gamma$, where $\sigma \in G, \alpha \in U$, and $\gamma \in\langle G, U\rangle$. Since $\varepsilon \alpha=\alpha$, where $\varepsilon$ is the identity in $G=G_{K}$, we may assume that $\eta=\sigma \alpha \gamma$. Thus, by Lemma 4.4, type ${ }_{\mu}(\alpha)=\operatorname{type}_{\mu}(\sigma \alpha)=\operatorname{type}_{\mu}(\eta)=\left(n_{1}, n_{2}, \ldots, n_{r} ; m\right)$. Also, $\eta=\theta \beta$, where $\theta \in\langle G, U\rangle$ and $\beta \in U$. (Note that $\beta$ cannot be followed by any element $\delta \in G$ since $\operatorname{im}(\eta)=L$ and $L \nsubseteq K$.) Then $L \subseteq \operatorname{im}(\beta)$, and so $\operatorname{im}(\beta)=L$ since $|L|=\operatorname{rank}(\beta)=r$.

Conversely, suppose that the set $U$ satisfies the given condition. Let $\gamma=\left(\begin{array}{l}C_{i} \\ y_{i}\end{array}\right) \in J_{r}$, and let $\left(n_{1}, n_{2}, \ldots, n_{r} ; m\right)$ be the $\mu$-type of $\gamma$ (so $n_{i}=\left|C_{i}\right|$ for every $i$ ) and $L=\operatorname{im}(\gamma)=\left\{y_{i}\right\}$. By the hypothesis, there is $\alpha=\left(\begin{array}{c}A_{i} \\ x_{i}\end{array}\right) \in U$ with type ${ }_{\mu}(\alpha)=\left(n_{1}, n_{2}, \ldots, n_{r} ; m\right)$. Then, for every $i,\left|A_{i}\right|=$ $\left|C_{i}\right|$, so there is a bijection $f_{i}: A_{i} \rightarrow C_{i}$. Define $\sigma: \bigcup_{1 \leq i \leq r} A_{i} \rightarrow K$ by $a \sigma=a f_{i}$ if $a \in A_{i}$, and extend $\sigma$ to $\bar{\sigma} \in G$. (Since $X$ is finite, Lemma 2.2 is also true if $|\bar{L}|=|K|$.) Then

$$
(\bar{\sigma})^{-1} \alpha \bar{\sigma}=\left(\begin{array}{c}
A_{i} \bar{\sigma} \\
x_{i} \bar{\sigma}
\end{array}\right)=\left(\begin{array}{c}
A_{i} f_{i} \\
x_{i} \bar{\sigma}
\end{array}\right)=\left(\begin{array}{c}
C_{i} \\
x_{i} \bar{\sigma}
\end{array}\right)
$$

Suppose $\left\{y_{i}\right\}_{1 \leq i \leq r} \subseteq K$. Define $\delta:\left\{x_{i} \bar{\sigma}\right\} \rightarrow K$ by $\left(x_{i} \bar{\sigma}\right) \delta=y_{i}$, and extend $\delta$ to $\bar{\delta} \in G$. Then $(\bar{\sigma})^{-1} \alpha \bar{\sigma} \bar{\delta}=\gamma$. Suppose $\left\{y_{i}\right\} \nsubseteq K$. Then, by the hypothesis, there is $\beta=\left(\begin{array}{c}B_{i} \\ y_{i}\end{array}\right) \in U$. Fix $b_{i} \in B_{i}$, define $\delta:\left\{x_{i} \bar{\sigma}\right\} \rightarrow K$ by $\left(x_{i} \bar{\sigma}\right) \delta=b_{i}$, and extend $\delta$ to $\bar{\delta} \in G$. Then $(\bar{\sigma})^{-1} \alpha \bar{\sigma} \bar{\delta} \beta=\gamma$. Hence $\gamma \in\langle G, U\rangle$, and so $J_{r} \subseteq\langle G, U\rangle$.

For positive integers $n$ and $r \leq n$, denote by $p_{r}(n)$ the number of partitions of $n$ with $r$ parts. For example, $(3,1,1)$ and $(2,2,1)$ are the only partitions of 5 with 3 parts, so $p_{3}(5)=2$. There is no known closed formula for calculating $p_{r}(n)$. For recursive formulas, see [14, Theorem 2.4.4].

Lemma 4.6. Let $\alpha \in P_{\mu}(X)$ with $\operatorname{rank}(\alpha)=r<v-1$. Then, there are $\varepsilon, \gamma \in P_{\mu}(X)$, both of rank $r+1$, such that $\alpha=\varepsilon \gamma$. 
Proof. Let $\alpha=\left(\begin{array}{c}A_{i} \\ x_{i}\end{array}\right)$ and fix $a_{i} \in A_{i}$. Choose $y \in X$ such that $y \notin x_{i} \mu$, for every $i, 1 \leq i \leq r$ (possible since $r<v-1$ and $|X / \mu|=v$ ), and note that $y \notin\left\{x_{1}, \ldots, x_{r}\right\}$.

Suppose $\left|A_{t}\right| \geq 3$, for some $t$, and let $b, c \in A_{t}$ with $b \neq c$ and $b, c \neq a_{t}$. Define $\varepsilon \in P_{\mu}(X)$, with $\operatorname{dom}(\varepsilon)=\operatorname{dom}(\alpha)$, by $k \varepsilon=a_{i}$ if $k \in A_{i}$ and $i \neq t, k \varepsilon=a_{t}$ if $k \in A_{t}$ and $k \neq b$, and $b \varepsilon=b$. Then $\operatorname{im}(\varepsilon)=\left\{a_{i}\right\}_{1 \leq i \leq r} \cup\{b\}$. Define $\gamma \in P_{\mu}(X)$, with $\operatorname{dom}(\gamma)=\operatorname{dom}(\alpha)$, by $k \gamma=x_{i}$ if $k \in A_{i}$ and $i \neq t, k \gamma=x_{t}$ if $k \in A_{t}$ and $k \neq c$, and $c \gamma=y$. Then $\operatorname{im}(\varepsilon)=\left\{x_{i}\right\}_{1 \leq i \leq r} \cup\{y\}$.

Suppose $\left|A_{s}\right|=\left|A_{t}\right|=2$, for some distinct $s, t$, say $A_{s}=\left\{a_{s}, b\right\}$ and $A_{t}=\left\{a_{t}, c\right\}$. Define $\varepsilon \in P_{\mu}(X)$, with $\operatorname{dom}(\varepsilon)=\operatorname{dom}(\alpha)$, by $k \varepsilon=a_{i}$ if $k \in A_{i}$ and $i \neq s, a_{s} \varepsilon=a_{s}$, and $b \varepsilon=b$. Define $\gamma \in P_{\mu}(X)$, with $\operatorname{dom}(\gamma)=\operatorname{dom}(\alpha)$, by $k \gamma=x_{i}$ if $k \in A_{i}$ and $i \neq t, a_{t} \gamma=x_{t}$, and $c \gamma=y$.

Suppose $\left|A_{s}\right|=2$, for some $s$, say $A_{s}=\left\{a_{s}, b\right\}$, and $\left|A_{i}\right|=1$ for every $i \neq s$. Since $r<v-1$, there is $c \in K$ such that $c \notin A_{i}$ for every $i$. Define $\varepsilon$ exactly as in the previous case. Define $\gamma \in P_{\mu}(X)$, with $\operatorname{dom}(\gamma)=\operatorname{dom}(\alpha) \cup c \mu$, by $k \gamma=x_{i}$ for all $k \in A_{i}$, and $c \gamma=y$.

Finally, suppose $\left|A_{i}\right|=1$ for every $i$. Since $r<v-1$, there are distinct $b, c \in K$ such that $b, c \notin A_{i}$ for every $i$. Define $\varepsilon \in P_{\mu}(X)$, with $\operatorname{dom}(\varepsilon)=\operatorname{dom}(\alpha) \cup b \mu$, by $k \varepsilon=a_{i}$ for every $k \in A_{i}$, and $b \varepsilon=b$. Define $\gamma$ exactly as in the previous case.

In all cases, $\operatorname{im}(\varepsilon)=\left\{a_{i}\right\}_{1 \leq i \leq r} \cup\{b\}$ (so $\left.\operatorname{rank}(\varepsilon)=r+1\right), \operatorname{im}(\gamma)=\left\{x_{i}\right\}_{1 \leq i \leq r} \cup\{y\}$ (so $\operatorname{rank}(\gamma)=r+1)$, and $\alpha=\varepsilon \gamma$.

Let $\left\{Q_{1}, Q_{2}, \ldots, Q_{v}\right\}$ denote the set of $\mu$-classes.

Theorem 4.7. Let $v \geq 3$ and let $I_{r}$ be the ideal of $P_{\mu}(X)$ consisting of all transformations of rank $\leq r$, where $1 \leq r<v$. Then

$$
\operatorname{rank}\left(G \cup I_{r}\right)=\max \left\{\sum_{1 \leq i_{1}<\ldots<i_{r} \leq v}\left|Q_{i_{1}}\right| \cdots\left|Q_{i_{r}}\right|-\left(\begin{array}{c}
v \\
r
\end{array}\right), \sum_{m=0}^{v-r} p_{r}(v-m)\right\}+2 .
$$

Proof. Let $s_{1}=\sum_{1 \leq i_{1}<\ldots<i_{r} \leq v}\left|Q_{i_{1}}\right| \cdots\left|Q_{i_{r}}\right|-\left(\begin{array}{l}v \\ r\end{array}\right)$ and $s_{2}=\sum_{m=0}^{v-r} p_{r}(v-m)$. Then $s_{1}$ is the number of partial transversals $L$ of $\mu$ such that $|L|=r$ and $L \nsubseteq K$, and $s_{2}$ is the number of $\mu$-types $\left(n_{1}, n_{2}, \ldots, n_{r} ; m\right)$ with $0 \leq m \leq v-r$.

Suppose $s_{1} \geq s_{2}$. Construct a set $U$ of transformations of rank $r$ as follows. For every $\mu$-type $\tau=\left(n_{1}, n_{2}, \ldots, n_{r} ; m\right)$, select $\alpha$ with type ${ }_{\mu}(\alpha)=\tau$ and add it to $U$. Since $s_{1} \geq s_{2}$, we can make these selections in such a way that every $\alpha \in U$ has image $L \nsubseteq K$ and any two distinct $\alpha_{1}, \alpha_{2} \in U$ have distinct images. At this point, there are $s_{1}-s_{2}$ partial transversals $L$ of $\mu$ such that $|L|=r, L \nsubseteq K$, and $L$ is not the image of any $\alpha \in U$. For each such an $L$, select an idempotent (or any element) $\varepsilon \in I_{r}$ with $\operatorname{im}(\varepsilon)=L$ and add it to $U$. Then $U$ consists of $s_{1}$ elements of rank $r$.

Suppose $s_{1}<s_{2}$. Construct a set $U$ of transformations of rank $r$ as follows. For every $\mu$-type $\tau=\left(n_{1}, n_{2}, \ldots, n_{r} ; m\right)$, select $\alpha$ with type ${ }_{\mu}(\alpha)=\tau$ and add it to $U$. Since $s_{1}<s_{2}$, we can make these selections in such a way that for every partial transversal $L$ of $\mu$ with $|L|=r$ and $L \nsubseteq K$, there is $\alpha \in U$ such that $\operatorname{im}(\alpha)=L$. Then $U$ consists of $s_{2}$ elements of rank $r$.

In either case, the $\mu$-types of elements of $U$ cover all $\mu$-types $\left(n_{1}, n_{2}, \ldots, n_{r} ; m\right)$ and the images of elements of $U$ cover all partial transversals $L$ of $\mu$ such that $|L|=r$ and $L \nsubseteq K$. Thus, by Proposition 4.5, $\langle G, U\rangle$ contains all elements of $I_{r}$ of rank $r$. Further, by Lemma 4.6, it also contains all elements of $I_{r}$ of rank $<r$. Hence $G \cup U$ generates $G \cup I_{r}$. Moreover, by Proposition 4.5, $U$ is a set of the smallest cardinality such that $G \cup U$ generates $G \cup I_{r}$.

The result now follows since $|U|=\max \left\{s_{1}, s_{2}\right\}$ and $G \cong \operatorname{Sym}(K)$, so it is generated by 2 elements. 
If $v \geq 1$ and $r=0$, then $I_{0}=\{0\}$, so $G \cup I_{0}$ has rank 3 if $v \geq 3$ (since then $G$ has rank 2), and it has rank 2 if $v \in\{1,2\}$ (since then $G$ has rank 1). If $v=2$ and $r=1$, then $G \cup I_{1}$ has rank $\max \left\{s_{1}, s_{2}\right\}+1$ (since then $G$ has rank 1 ).

The following result is a special case of [4, Theorem 4.4].

Lemma 4.8. Let $J_{r}$ be the $\mathcal{J}$-class in $P_{\mu}(X)$ consisting of all transformations of rank $r$, where $0 \leq r \leq$ $v$. Then:

(1) $J_{r}$ has $S(v+1, r+1) \mathcal{R}$-classes and $\sum_{1 \leq i_{1}<\ldots<i_{r} \leq v}\left|Q_{i_{1}}\right| \cdots\left|Q_{i_{r}}\right| \mathcal{L}$-classes;

(2) $J_{r}$ has $r ! S(v+1, r+1) \sum_{1 \leq i_{1}<\ldots<i_{r} \leq v}\left|Q_{i_{1}}\right| \cdots\left|Q_{i_{r}}\right|$ elements.

Recall that $J_{v}$ is the top $\mathcal{J}$-class of $P_{\mu}(X)$. A semigroup $S$ is called a right group if $S \cong G \times E$, where $G$ is a group and $E$ is a right zero semigroup [7, Exercise 6].

Proposition 4.9. Let $v \geq 1$. Then:

(1) $J_{v}$ is a right group;

(2) if $\mu \neq \mathrm{id}_{X}$, then $\operatorname{rank} J_{v}=\left|Q_{1}\right| \cdots\left|Q_{v}\right|$.

Proof. Recall that $J_{v}$ is the union of groups $G_{M}$, where $M$ ranges over all transversals of $\mu$. Fix one of these groups, say $G=G_{K}$, and let $E$ be the set of idempotents in $J_{v}$. Note each element of $E$ is the identity of some group $G_{M}$, and that for all $\varepsilon \in E$ and $\beta \in J_{v}, \varepsilon \beta=\beta$. Thus $E$ is a right zero semigroup. Define $f: G \times E \rightarrow J_{v}$ by $(\alpha, \varepsilon) f=\alpha \varepsilon$. The function $f$ is a homomorphism, since for all $(\alpha, \varepsilon),(\beta, \xi) \in G \times E$,

$$
((\alpha, \varepsilon)(\beta, \xi)) f=(\alpha \beta, \varepsilon \xi) f=(\alpha \beta, \xi) f=\alpha \beta \xi=\alpha(\varepsilon \beta) \xi=((\alpha, \varepsilon) f)((\beta, \xi) f) .
$$

Let $(\alpha, \varepsilon),(\beta, \xi) \in G \times E$ with $\alpha \varepsilon=\beta \xi$. Then $\operatorname{im}(\varepsilon)=\operatorname{im}(\xi)$, which implies $\varepsilon=\xi$ since an idempotent in $J_{v}$ is completely determined by its image. Let $x \in X$. Then $(x \alpha) \varepsilon=(x \beta) \xi=(x \beta) \varepsilon$, and so $x \alpha$ and $x \beta$ are in the same $\mu$-class (since $\operatorname{ker}(\varepsilon)=\mu$ ). Thus, since $\operatorname{im}(\alpha)=\operatorname{im}(\beta)=K$ and $K$ is a transversal of $\mu$, it follows that $x \alpha=x \beta$. Hence $(\alpha, \varepsilon)=(\beta, \xi)$, so $f$ is injective. Thus, it is also surjective since $G \times E$ and $J_{v}$ are finite semigroups of the same size. (Indeed, $|G|=r !,|E|=\left|Q_{1}\right| \cdots\left|Q_{v}\right|$, and $\left|J_{v}\right|=r !\left|Q_{1}\right| \cdots\left|Q_{v}\right|$ by Lemma 4.8.) Hence $f$ is an isomorphism, which proves (1).

If $\mu \neq \mathrm{id}_{X}$, then $J_{v}$ satisfies the hypotheses of Lemma 4.2. By Lemma 4.8, $J_{v}$ has one $\mathcal{R}$-class and $\left|Q_{1}\right| \cdots\left|Q_{v}\right| \mathcal{L}$-classes, so (2) follows.

If $\mu=\mathrm{id}_{X}$, then $J_{v}=\operatorname{Sym}(X)$, and so rank $J_{v}=2$ if $|X| \geq 3$, and rank $J_{v}=1$ if $|X| \leq 2$.

Theorem 4.10. Let $v \geq 2$ and let $I_{r}$ be the ideal of $P_{\mu}(X)$ consisting of all elements of rank $\leq r$, where $1 \leq r<v$. Then

$$
\operatorname{rank}\left(J_{v} \cup I_{r}\right)=\sum_{m=0}^{v-r} p_{r}(v-m)+\operatorname{rank} J_{v},
$$

where $\operatorname{rank} J_{v}=\left|Q_{1}\right| \cdot\left|Q_{2}\right| \cdots\left|Q_{v}\right|$ if $\mu \neq \mathrm{id}_{X}$, rank $J_{v}=2$ if $\mu=\mathrm{id}_{X}$ and $|X| \geq 3$, and $\operatorname{rank} J_{v}=1$ if $|X|=2$.

Proof. Let $A$ be any set of generators of $J_{v} \cup I_{r}$. Let $\left(n_{1}, n_{2}, \ldots, n_{r} ; m\right)$ be a $\mu$-type. Since $A$ generates $J_{v} \cup I_{r}$, there is $\eta \in\langle A\rangle$ such that $\operatorname{type}_{\mu}(\eta)=\left(n_{1}, n_{2}, \ldots, n_{r} ; m\right)$. Since $\operatorname{rank}(\eta)=r$, we have $\eta=\sigma \alpha \gamma$ or $\eta=\alpha \gamma$, where $\sigma \in J_{v}, \alpha \in A$ with $\operatorname{rank}(\alpha)=r$, and $\gamma \in J_{v} \cup J_{r}$. Since $\varepsilon \alpha=$ $\alpha$ for any idempotent $\varepsilon \in J_{v}$, we may assume that $\eta=\sigma \alpha \gamma$. Thus, by Lemma 4.4, $\operatorname{type}_{\mu}(\alpha)=$ 
$\operatorname{type}_{\mu}(\sigma \alpha)=\operatorname{type}_{\mu}(\eta)=\left(n_{1}, n_{2}, \ldots, n_{r} ; m\right)$. Hence for every $\mu$-type $\tau=\left(n_{1}, n_{2}, \ldots, n_{r} ; m\right), A$ contains an element $\alpha$ with type ${ }_{\mu}(\alpha)=\tau$. Since $A$ must also contain a generating set of $J_{v}$ and $\sum_{m=0}^{v-r} p_{r}(v-m)$ is the number of $\mu$-types $\left(n_{1}, n_{2}, \ldots, n_{r} ; m\right)$, we have $|A| \geq \sum_{m=0}^{v-r} p_{r}(v-m)+$ rank $J_{v}$, and so $\operatorname{rank}\left(J_{v} \cup I_{r}\right) \geq \sum_{m=0}^{v-r} p_{r}(v-m)+\operatorname{rank} J_{v}$.

We will now construct a set $A$ of generators of $J_{v} \cup I_{r}$ with exactly $\sum_{m=0}^{v-r} p_{r}(v-m)+\operatorname{rank} J_{v}$ elements. Begin with $A$ being a set of generators of $J_{v}$ of the smallest cardinality. Then for every $\mu$-type $\tau=\left(n_{1}, n_{2}, \ldots, n_{r} ; m\right)$, select $\alpha$ with type ${ }_{\mu}(\alpha)=\tau$ and add it to $A$. Let $L=\left\{m_{1}, m_{2}, \ldots, m_{r}\right\}$ be any partial transversal of $\mu$ of size $r$. Then $L \subseteq M$ for some transversal $M$ of $\mu$. Select any $\alpha=\left(\begin{array}{c}A_{i} \\ x_{i}\end{array}\right) \in A$ of rank $r$. Define $\sigma:\left\{x_{i}\right\} \rightarrow M$ by $x_{i} \sigma=m_{i}$ and extend $\sigma$ to $\bar{\sigma} \in G_{M}$ (possible by Lemma 2.2 applied to $\left.G_{M}\right)$. Then

$$
(\bar{\sigma})^{-1} \alpha \bar{\sigma}=\left(\begin{array}{c}
A_{i} \bar{\sigma} \\
x_{i} \bar{\sigma}
\end{array}\right)=\left(\begin{array}{c}
A_{i} \bar{\sigma} \\
m_{i}
\end{array}\right) .
$$

Thus $(\bar{\sigma})^{-1} \alpha \bar{\sigma} \in\langle A\rangle$ and $\operatorname{im}\left((\bar{\sigma})^{-1} \alpha \bar{\sigma}\right)=L$. Hence the $\mu$-types of elements of $\langle A\rangle$ cover all $\mu$-types $\left(n_{1}, n_{2}, \ldots, n_{r} ; m\right)$ and the images of elements of $\langle A\rangle$ cover all partial transversals $L$ of $\mu$ such that $|L|=r$. Thus, by Proposition 4.5, $\langle G \cup U\rangle$, where $U=\{\alpha \in\langle A\rangle: \operatorname{rank}(\alpha)=r\}$, contains all elements of $I_{r}$ of rank $r$. Further, by Lemma 4.6, it also contains all elements of $I_{r}$ of rank $<r$. Hence $\langle A\rangle=J_{v} \cup I_{r}$ since $J_{v} \subseteq\langle A\rangle$ and $G \cup U \subseteq\langle A\rangle$. The cardinality of $A$ is $\sum_{m=0}^{v-r} p_{r}(v-m)+\operatorname{rank} J_{v}$ by the construction, so it follows that $\operatorname{rank}\left(J_{v} \cup I_{r}\right) \leq \sum_{m=0}^{v-r} p_{r}(v-m)+\operatorname{rank} J_{v}$.

Hence $\operatorname{rank}\left(J_{v} \cup I_{r}\right)=\sum_{m=0}^{v-r} p_{r}(v-m)+\operatorname{rank} J_{v}$. Finally, the statements about the rank of $J_{v}$ are true by Proposition 4.9 .

If $r=0$, then $I_{0}=\{0\}$, so $\operatorname{rank}\left(J_{v} \cup I_{0}\right)=\operatorname{rank} J_{v}+1$.

Since $P_{\mu}(X)=J_{v} \cup I_{v-1}$ and $\sum_{m=0}^{v-(v-1)} p_{v-1}(v-m)=p_{v-1}(v)+p_{v-1}(v-1)=2$, we have $\operatorname{rank} P_{\mu}(X)=\operatorname{rank} J_{v}+2$ if $v \geq 2$. If $v=1$, then $\operatorname{rank} P_{\mu}(X)=\operatorname{rank} J_{v}+1=\left|Q_{1}\right|+1=n+2$, where $n=|X|$. These facts can also be deduced from the more general [4, Theorem 4.5].

The result for each proper ideal $I_{r}$ of $P_{\mu}(X)$ follows from Lemma 4.2.

Corollary 4.11. Let $v \geq 2$ and let $I_{r}$ be the ideal of $P_{\mu}(X)$ consisting of all transformations of rank $\leq r$, where $1 \leq r<v$. Then

$$
\operatorname{rank} I_{r}=\max \left\{\sum_{1 \leq i_{1}<\ldots<i_{r} \leq v}\left|Q_{i_{1}}\right| \cdots\left|Q_{i_{r}}\right|, S(v+1, r+1)\right\} .
$$

Proof. The top $\mathcal{J}$-class $J_{r}$ of $I_{r}$ satisfies the hypotheses of Lemma 4.2 by Theorem 2.6, Lemma 4.6, and the fact that $v \geq 2$ and $1 \leq r<v$. Thus the result follows by Lemma 4.8.

If $v \geq 1$ and $r=0$, Then $I_{r}=\{0\}$ has rank 1 .

The results and proofs of this section carry over to the semigroup $T_{\mu}(X)=P_{\mu}(X) \cap T(X)$, where $T(X)$ is the semigroup of full transformations on $X$. The only differences are that $T_{\mu}(X)$ has no ideal $I_{0}$ and each element of $T_{\mu}(X)$ has deficit 0 , so the sum $\sum_{m=0}^{v-r} p_{r}(v-m)$ reduces to $p_{r}(v)$. Note that the $\mathcal{J}$-classes $J_{v}$ in $P_{\mu}(X)$ and $T_{\mu}(X)$ are the same. 
Theorem 4.12. Let $v \geq 3$ and let $I_{r}$ be the ideal of $T_{\mu}(X)$ consisting of all elements of rank $\leq r$, where $1 \leq r<v$. Then

$$
\begin{aligned}
\operatorname{rank}\left(G \cup I_{r}\right) & =\max \left\{\sum_{1 \leq i_{1}<\ldots<i_{r} \leq v}\left|Q_{i_{1}}\right| \cdots\left|Q_{i_{r}}\right|-\left(\begin{array}{c}
v \\
r
\end{array}\right), p_{r}(v)\right\}+2, \\
\operatorname{rank}\left(J_{v} \cup I_{r}\right) & =p_{r}(v)+\operatorname{rank} J_{v}, \\
\operatorname{rank} I_{r} & =\max \left\{\sum_{1 \leq i_{1}<\ldots<i_{r} \leq v}\left|Q_{i_{1}}\right| \cdots\left|Q_{i_{r}}\right|, S(v, r)\right\} .
\end{aligned}
$$

Consequently, $\operatorname{rank} T_{\mu}(X)=\operatorname{rank} J_{v}+1$

The result for $\operatorname{rank} T_{\mu}(X)$ can also be deduced from the more general [5, Theorem 5.18].

Acknowledgment. We are grateful to the referee for a very careful reading of the paper.

\section{References}

[1] J. André, J. Araújo, and P.J. Cameron, The classification of partition homogeneous groups with applications to semigroup theory, J. Algebra 452 (2016), 288-310.

[2] J. Araújo and J. Konieczny, Semigroups of transformations preserving an equivalence relation and a cross-section, Comm. Algebra 32 (2004), 1917-1935.

[3] A.H. Clifford and G.B. Preston, The Algebraic Theory of Semigroups, Mathematical Surveys, No. 7, American Mathematical Society, Providence, Rhode Island, 1964 (Vol. I) and 1967 (Vol. II).

[4] I. Dolinka, I. Durdev, J. East, P. Honyam, K. Sangkhanan, J. Sanwong, and W. Sommanee, Sandwich semigroups in locally small categories II: transformations, Algebra Universalis 79 (2018), Art. 76, 53 pp.

[5] I. Dolinka and J. East, Variants of finite full transformation semigroups, Int. J. Algebra Comput. 25 (2015), 1187-1222.

[6] R.D. Gray, The minimal number of generators of a finite semigroup, Semigroup Forum 89 (2014), $135-154$.

[7] J.M. Howie, Fundamentals of Semigroup Theory, Oxford University Press, New York, 1995.

[8] K. Hrbacek and T. Jech, Introduction to Set Theory, Third Edition, Taylor \& Francis, New York, 1999.

[9] S. Madhavan, On right normal right inverse semigroups, Semigroup Forum 12 (1976), 333-339.

[10] S. Mendes-Gonçalves and R.P. Sullivan, Semigroups of transformations restricted by an equivalence, Cent. Eur. J. Math. 8 (2010), 1120-1131.

[11] H. Pei, Regularity and Green's relations for semigroups of transformations that preserve an equivalence, Comm. Algebra 33 (2005), 109-118. 
[12] H. Pei and H. Zhou, Semigroups of partial transformations preserving an equivalence relation, Adv. Math. (China) 38 (2009), 103-116.

[13] M. Petrich, Inverse Semigroups, John Wiley \& Sons, New York, 1984.

[14] H.J. Straight, Combinatorics: An Invitation, Brooks/Cole Publishing Co., Pacific Grove, CA, 1993. 